Article

\title{
Green and Facile Synthesis of Dendritic and Branched Gold Nanoparticles by Gelatin and Investigation of Their Biocompatibility on Fibroblast Cells
}

\author{
Quoc Khuong Vo ${ }^{1}$, My Nuong Nguyen Thi ${ }^{2}$, Phuong Phong Nguyen Thi ${ }^{1, *}$ and \\ Duy Trinh Nguyen ${ }^{3,4, * \mathbb{D}}$ \\ 1 Faculty of Chemistry, Ho Chi Minh City University of Science, Vietnam National University-Ho Chi Minh \\ City, Ho Chi Minh City 700000, Vietnam; vqkhuong@hcmus.edu.vn \\ 2 Faculty of Biology and Biotechnology, Ho Chi Minh City University of Science, Vietnam National \\ University-Ho Chi Minh City, Ho Chi Minh City 700000, Vietnam; ntmnuong@hcmus.edu.vn \\ 3 NTT Hi-Tech Institute, Nguyen Tat Thanh University, Ho Chi Minh City 700000, Vietnam \\ 4 Center of Excellence for Green Energy and Environmental Nanomaterials, Nguyen Tat Thanh University, \\ Ho Chi Minh City 700000, Vietnam \\ * Correspondence: ntpphong@hcmus.edu.vn (P.P.N.T.); ndtrinh@ntt.edu.vn (D.T.N.)
}

Received: 3 August 2019; Accepted: 3 September 2019; Published: 18 September 2019

\begin{abstract}
In this work, gold nanostar (AuNPs) and gold nanodendrites were synthesized by one-pot and environmentally friendly approach in the presence of gelatin. Influence of gelatin concentrations and reaction conditions on the growth of branched (AuNPs) were investigated further. Interestingly, the conversion of morphology between dendritic and branched nanostructure can be attained by changing the $\mathrm{pH}$ value of gelatin solution. The role of gelatin as a protecting agent through the electrostatic and steric interaction was also revealed. Branched nanoparticles were characterized by surface plasmon resonance spectroscopy (SPR), transmission electron microscopy (TEM), XRD, dynamic light scattering (DLS) and zeta-potential. The chemical interaction of gelatin with branched gold nanoparticles was analyzed by Fourier transform infrared spectroscopy (FT-IT) technique. Ultraviolet visible spectroscopy results indicated the formation of branched gold nanoparticles with the maximum surface plasmon resonance peak at 575-702 $\mathrm{nm}$. The structure of both nanodendrites and nanostars were determined by TEM. The crystal sizes of nano-star ranged from 42 to $55 \mathrm{~nm}$ and the nanodendrites sizes were about 75-112 nm. Based on the characterizations, a growth mechanism could be proposed to explain morphology evolutions of branched AuNPs. Moreover, the branched AuNPs is high viability at $100 \mu \mathrm{g} / \mathrm{mL}$ concentration when performed by the SRB assay with human foreskin fibroblast cells.
\end{abstract}

Keywords: nanodendrites; nanostar; fibroblast cells; gelatin; one-pot synthesis

\section{Introduction}

In recent years, multi-branched gold nanoparticles (AuNPs) have gained focus as the most promising nanomaterial for biomedical applications due to their unique properties such as surface functionalization and biocompatibility, which highly depend on structural features of the nanoparticles [1]. The use of diverse gold nanostructures in drugs delivery and site-specific cell binding have been effectively studied. For instance, some bioactive molecules such as amino acid, peptide or protein could be specifically conjugated to the multi-branched AuNPs, or the rod-shaped nanoparticles have been researched to enhance higher cellular uptake [2,3]. Thus, to expand the array of application, many synthetic methods are focused on tuning size [4], shape [3,5], composition, assembly [6] and encapsulation of AuNPs [7]. However, these synthetic methods could rely on organic solvents or toxic 
ingredients which limit the application of AuNPs in biological and medical fields $[3,8]$. This calls for the development of green preparation routes for the controlled synthesis of multi-branched AuNPs. Pathways for the synthesis of multi-branched gold nanoparticles consist of seed-mediated and one-pot synthesis. The former method is capable of producing AuNPs with tunable morphology. However, the synthesis of the branched gold nanoparticles commonly requires cetyl trimethyl ammonium bromide (CTAB) to control the growth of crystal face [9]. This surfactant is toxic and thus limiting AuNPs synthesized in biological applications [10]. Therefore, to prepare a biocompatible system containing multi-branched AuNPs with non-toxic reducing and stabilizing agents, another technique that is environmentally friendly is required.

As a natural polymer, gelatin has been recently used as a 'green' stabilizing agent to prepare AuNPs because of its non-toxicity biocompatibility $[8,11]$ and biodegradable properties [8]. This natural polymer can be adsorbed effectively onto the particle surface and prevent the nanoparticles from aggregating. The interaction between gelatin and AuNPs has been proposed through its abundant functional groups.

Recently, though gelatin has been widely incorporated into many modified AuNPs materials such as nanocarriers for drug delivery or engineering tissue [11], the structure-directing effect of gelatin is rarely investigated. Due to the structural transformation in different conditions, gelatin could be an appropriate component for controlling the growth of gold nanoparticles. Furthermore, this hydrophilic coating allows better dispersion of the synthesized AuNPs, which could be suitable for testing in biological experiments. Suarasan et al. have reported the role of gelatin as a stabilizer in the green synthesis of gold nanoparticles. The obtained gelatin coated AuNPs was tested for stability and biocompatibility with Osteoblast cells. Additionally, the interaction between gelatin and AuNPs were also revealed through the steric and electrostatic mechanisms in aqueous media [6]. The distinct stages in the synthesis of AuNPs were investigated by Yi-Chen Wang and co-workers using the mixture of $\mathrm{HAuCl}_{4} / \mathrm{HEPES}$ and gelatin solution. Based on their proposed mechanism, it was suggested that the shape and size of AuNPs were strongly dependent on the nucleation time and heating temperatures [12]. The stability of AuNPs at high ionic strength solution was greatly improved in the presence of gelatin, as reported by Sorina Suarasan et al. The obtained gelatin@AuNPs was demonstrated as the effective substrate for surface-enhanced Raman scattering (SERS) detection of Rose Bengal fluorophore [13]. By using an in-situ synthesis method with sodium citrate, the size of AuNPs was also shown to be controllable by varying concentration of gelatin solution [14].

The formation of AuNPs is substantially influenced by not only the reducing and stabilizing agents, but also by the experimental conditions. In this work, we propose a direct approach to prepare AuNPs with multi-branched morphology using gelatin as a protecting agent. Unlike previous procedures, one-pot synthetic method is developed to obtain highly branched gold nanocrystals. The advantage of this method is the absence of template or surfactants, most of which are insufficiently environmentally-friendly to be applied in biomedical engineering, medicine and biotechnology. In addition, our method requires no hazardous chemicals and occurs in aqueous solution. The multi-branched structures of AuNPs are controlled by changing the experimental conditions such as temperature, $\mathrm{pH}$ value and stabilizing factors. The synthesis reaction proceeded in about $45 \mathrm{~min}$, and then the branched AuNPs was formed without heating. To the best of our knowledge, this preparation process for multi-branched AuNPs requires the lowest temperature. Finally, the stability of gelatin at different $\mathrm{pH}$ values was also investigated to propose mechanism growth of branched and dendritic structures.

\section{Experimental}

\subsection{Materials}

Gelatin (Type A) from porcine skin was purchased from Sigma-Aldrich (St. Louis, MO, USA). Tetrachloroauric (III) acid trihydrate $\left(\mathrm{HAuCl}_{4} \cdot 3 \mathrm{H}_{2} \mathrm{O}, 99.99 \%\right)$, acetic acid and trichloroacetic acid were 
purchased from Merck (Darmstadt, Germany). Ascorbic acid $\left(\mathrm{C}_{6} \mathrm{H}_{8} \mathrm{O}_{6}\right)$, trisodium citrate dihydrate $\left(\mathrm{C}_{6} \mathrm{H}_{5} \mathrm{Na}_{3} \mathrm{O}_{7} \cdot 2 \mathrm{H}_{2} \mathrm{O}\right)$, anti-vimentin/anti-cytokeratin 19 anti-bodies, collagenase and sulforhodamine B (SRB) were purchased from Sigma-Aldrich. Dulbecco's Modified Eagle Medium (DMEM) and Nutrient mixture F-12 were purchased from Gibco (Dún Laoghaire, Ireland). Tris base solution was purchased from Promega (Madison, WI, USA). Camptothecin was purchased from Calbiochem (Darmstadt, Germany) and used as received. All chemical reagents and solvents were of analytical grade, and aqueous solutions were prepared with deionized water (conductivity was below $4.3 \mu \mathrm{S} / \mathrm{cm}$ ).

\subsection{Preparation of AuNPs}

Prior use, glassware was cleaned using aqua regia $\left(1: 3, \mathrm{HNO}_{3}: \mathrm{HCl}, \mathrm{v} / \mathrm{v}\right)$, followed by rinsing with deionized water. To prepare colloidal gold solution, $\mathrm{HAuCl}_{4}$ underwent reduction by ascorbic acid in the presence of gelatin. Prior to synthesis, the gelatin powder was freshly dissolved in ultrapure water and vigorously stirred for $10 \mathrm{~min}$ at $50{ }^{\circ} \mathrm{C}$ to avoid degradation of gelatin during storage. In a typical procedure, $100 \mu \mathrm{L}$ of $25 \mathrm{mM} \mathrm{HAuCl}_{4}$ solution was added into $1.0 \mathrm{~mL}$ gelatin $(0.1 \%, w / v)$ solution at $40^{\circ} \mathrm{C}$ of temperature, and then the mixture was magnetically stirred at $700 \mathrm{rpm}$ for $45 \mathrm{~min}$. Subsequently, an aqueous solution of ascorbic acid $(0.5 \mathrm{~mL}, 0.1 \mathrm{M})$ was added into reaction mixture. DI water was finally added to adjust the total volume to $10 \mathrm{~mL}$. During this time, the color of the reaction solution changed from transparent to pink, purple-blue and to deep blue, which is indicative of branched AuNPs. The influences of the gelatin concentrations and different experimental conditions on the synthesis of AuNPs were investigated. Additionally, shapes and sizes of the obtained gold nanoparticles were experimentally altered by varying the $\mathrm{pH}$ value. The certain amount of $1.00 \mathrm{M}$ $\mathrm{NaOH}$ was added to change the $\mathrm{pH}$ value of the solution.

\subsection{Characterization}

The branched AuNPs colloidal solutions were characterized by UV-Vis adsorption spectroscopy on a UV-Vis-NIR-V670 spectrophotometer (JASCO, Japan), operated between 300 and $800 \mathrm{~nm}$ with scanning rate $200 \mathrm{~nm}$ per minute using quartz cuvettes (1-cm path length). FT-IR analysis was performed on a Tensor 27-Bruker spectrometer. $3.00 \mathrm{~mL}$ of analysis sample was mixed with $\mathrm{KBr}$ at ratio $2-5 \%$, scanning wavenumber from $400-4000 \mathrm{~cm}^{-1}$. The XRD pattern was recorded using D8 Advance-Bruker, Germany with $\mathrm{Cu}-\mathrm{K} \alpha$ radiation in the $2 \theta$ range from $30^{\circ}$ to $80^{\circ}$, operated at $40 \mathrm{kV}$. As-synthesized AuNPs colloids were centrifuged and subsequently dried under vacuum at room temperature to obtain dry powders. These powder samples were placed on glass and subjected to X-Ray, and then characterized at scanning rate of $0.02^{\circ}$ per second. Wide-angle XRD showed a face-centered cubic structure, five peaks are assigned to (111), (200), (220), (311), and (222) facets. The morphology of nanoparticles was investigated by Transmission Electron Microscopy (TEM) using JEM-1400, Japan. The samples were dropped small amount of AuNPs colloidal solution onto the carbon-copper grids (300-mesh, Ted Pella, Inc., Redding, CA, USA) and evaporated at room temperature. The average diameter and size distribution of branched AuNPs were determined statistically with the mean value of 200 particles in several chosen areas in microphotographs.

\subsection{Primary Fibroblast Culture}

Human foreskin fibroblast cells were obtained from a healthy tissue donor at Department of Andrology in Binh Dan Hospital, Ho Chi Minh City, Vietnam. This work was ethically approved by the Vietnam National University, Ho Chi Minh City. Informed consent forms of tissue donors were obtained before the interview, physical examination and sample collection commenced. Tissues were collected and used for research purposes only. Tissues after collection were stored in PBS, and then split into small pieces with dimensions of $2-3 \mathrm{~mm} \times 5 \mathrm{~mm}$. Incubation was carried out with $0.2 \%$ $(w / v)$ collagenase (Sigma) at $37^{\circ} \mathrm{C}$ for $2 \mathrm{~h}$. After being filtered through a cell strainer of about $70 \mu \mathrm{m}$ diameter, the cell suspension was centrifuged at 200x $g, 10 \mathrm{~min}$ and then resuspended in Dulbecco's Modified Eagle Medium: nutrient mixture F12 (DMEM/F12) (Gibco). Cell culture was performed in 
the DMEM/F12 media supplemented with 10\% (v/v) FBS, $20 \mathrm{mM}$ HEPES, $0.025 \mu \mathrm{g} / \mathrm{mL}$ amphotericin B, $100 \mathrm{IU} / \mathrm{mL}$ penicillin $\mathrm{G}, 100 \mu \mathrm{g} / \mathrm{mL}$ streptomycin at $37{ }^{\circ} \mathrm{C}, 5 \% \mathrm{CO}_{2}$. To allow identification of primary fibroblasts, anti-vimentin/anti-cytokeratin 19 anti-bodies (Sigma) stain was used and cell morphology was observed.

\subsection{SRB Assay}

The cytotoxicity of branched AuNPs was estimated by applying an SRB assay. First, 96-well plates were inoculated with cells at a culture density of 10,000 cells per well for $24 \mathrm{~h}$, followed by AuNPs treatment at different concentrations for $48 \mathrm{~h}$. Before washing and staining, a cold $50 \%(w / v)$ solution of trichloroacetic acid (Merck) was used to maintain the total protein of treated cells constant for 1-3 $\mathrm{h}$. Washing and staining were carried out with $0.2 \%(w / v)$ Sulforhodamine B (Sigma) for 20 min in which the dye was first washed five times with $1 \%$ acetic acid (Merck), followed by dissolution in $10 \mathrm{mM}$ Tris base solution (Promega). A 96-well micro titer plate reader (Synergy HT, Biotek Instruments) was used to measure the absorbance at two wavelengths of $492 \mathrm{~nm}$ and $620 \mathrm{~nm}$. Each experiment was conducted in triplicate to produce the mean result and standard deviation. The growth inhibitor value (Inh \%) was calculated as follows: Inh $\%=(1-[\mathrm{ODt} / \mathrm{Odc}] \times 100) \%$ where ODt and ODc denoted the optical density value of the test sample and the control sample, respectively. The positive control sample was camptothecin (Calbiochem).

\section{Result and Discussion}

\subsection{Influence of Gelatin Concentration}

The spherical AuNPs could be achieved with synthesis routes involving only either ascorbic acid or gelatin [13-15]. However, when being fabricated with the mixture of ascorbic acid and gelatin in an appropriate condition, produced AuNPs is highly branched. Furthermore, the presence of $\mathrm{NaOH}$ solution could play an important role in branched particle formation through enhanced the reduction ability of ascorbic acid [16]. Besides, the influence of gelatin on the evolution of particles shape without template was not clearly discussed. UV-Vis absorption spectra of Au colloidal solutions obtaining at different concentrations of gelatin $(0,0.5,1.0,1.5,2.0$ and 2.5\%) were examined, as shown in Figure 1 and Table 1. Without using of gelatin (curve a), a red colloid was obtained with maximum absorbance peak located at $532 \mathrm{~nm}$, indicated the optical signature for the spherical AuNPs in solution [17]. As the concentration of gelatin was increased to $2.5 \%(w / v)$, a significant red shift of surface plasmon resonance (SPR) band from 532 to $576 \mathrm{~nm}$ was recorded. The color of colloidal gold solution rapidly changed from wine red, red-purple and dark blue. Additionally, the new emerging band at $576 \mathrm{~nm}$ also revealed the transverse plasmon resonance of the tips, which was typically observed in branched structure of AuNPs [18]. From the UV-Vis results, the intensity of absorbance peak gradually increased from 1.091 to 1.188 , corresponded to the increase of gelatin concentration (from 0.5 to $2.0 \% w / v$ ) and the increase of the gold nanoparticles in colloidal solution. In contrast, as the gelatin concentration was $2.5 \%$, the decrease of intensity of absorbance peak could be caused by the decrease of the formed AuNPs. Corresponding TEM results in Figure 2 could also confirm the formation of branched AuNPs. The evolution of morphology from quasi-spherical (images 2-a) to branched nanoparticles (images 2-b) was observed when increased the concentration of gelatin. The average diameter of $92.5 \mathrm{~nm}$ was measured by TEM (Figure $2 \mathrm{~b}$ ) with the concentration of gelatin at $2.5 \%(w / v)$, which was consistent with the UV-Vis results. In fact, as the diffusion of particles through the solution significantly decreases due to the increasing of gelatin densification, the possibility of a particle reaching the crystal surface is also restricted. Thus, the branched structure is specially formed under diffusion-limited growth [19]. Otherwise, a higher hydrodynamic diameter of $117.3 \mathrm{~nm}$ was observed from dynamic light scattering (DLS) data, providing more information about the protecting layer of gelatin on the surface of nanoparticles. According to above results, the presence of gelatin can play both of protecting and growth controlling roles in the formation of branched particles. 


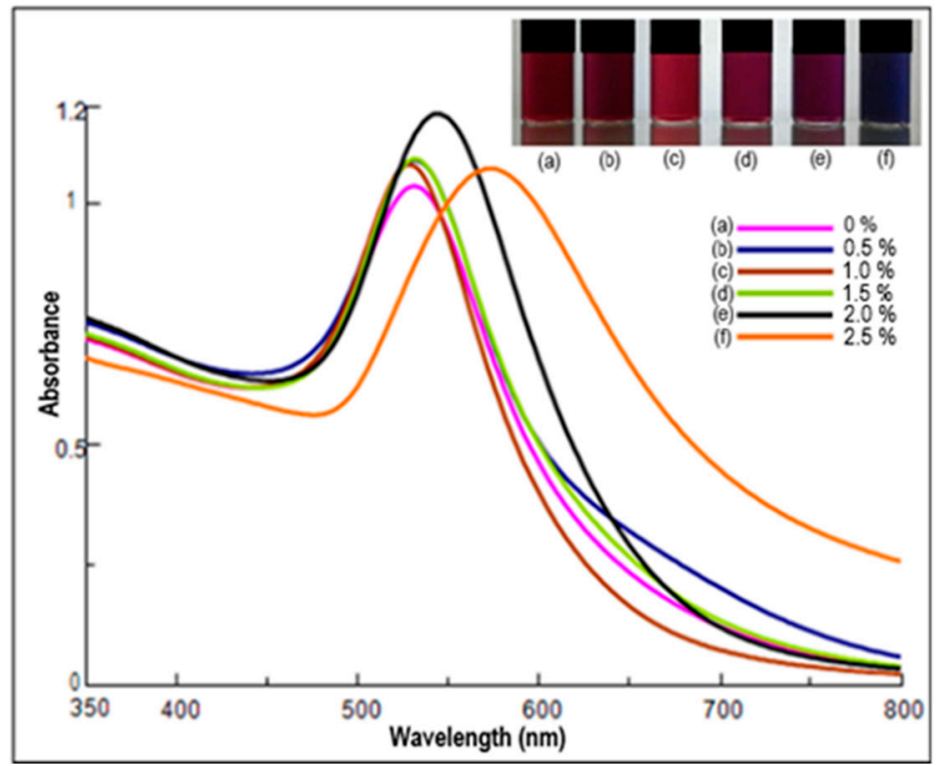

Figure 1. UV-Vis adsorption spectra of branched gold nanoparticles (AuNPs) synthesized by gelatin with different concentrations of (a) $0,(\mathbf{b}) 0.5$, (c) 1.0 , (d) 1.5 , (e) 2.0, (f) $2.5 \%(w / v)$.

Table 1. UV-Vis results of branched AuNPs synthesized by gelatin with different concentrations of gelatin.

\begin{tabular}{llll}
\hline Sample & $\begin{array}{l}\text { Gelatin } \\
\mathbf{C} \%(w / v)\end{array}$ & $\lambda \max (\mathbf{n m})$ & Absorbance \\
\hline G1 & 0 & 532 & 1.037 \\
\hline G2 & 0.5 & 532 & 1.091 \\
\hline G3 & 1.0 & 528 & 1.082 \\
\hline G4 & 1.5 & 532 & 1.094 \\
\hline G5 & 2.0 & 546 & 1.188 \\
\hline G6 & 2.5 & 576 & 1.074 \\
\hline
\end{tabular}

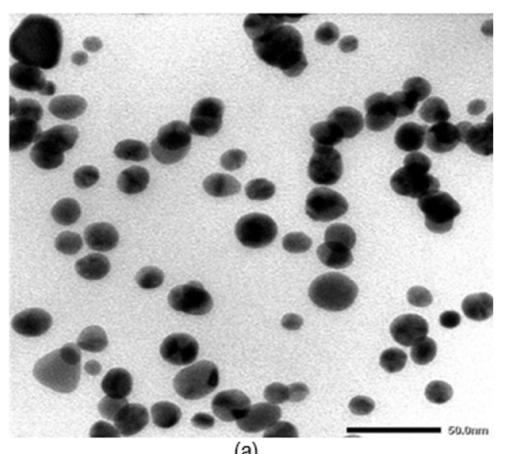

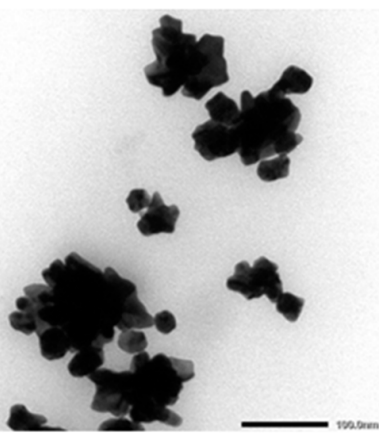

(b)

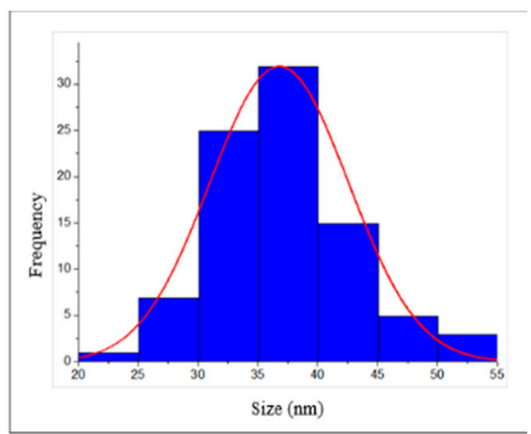

(c)

Figure 2. Representative TEM images of synthesized AuNPs with (a) low gelatin concentration (1.0\% $w / v)($ scale $50 \mathrm{~nm}),(\mathbf{b})$ high gelatin concentration $(2.5 \% w / v)($ scale $100 \mathrm{~nm})$ and (c) histogram of particle size distribution of synthesized AuNPs with low gelatin concentration $(1.0 \% w / v)$.

\subsection{Influence of Reaction Temperature}

The growth rate of AuNPs was extremely dependent on the temperature reaction, which also determined the viscosity and stability of gelatin on the colloidal solution [20]. However, if the synthesis of AuNPs was carried out at elevated temperature $\left(80^{\circ} \mathrm{C}\right)$, gelatin would be more degradable in the 
reaction solution. According to the previous reports [20,21], the conformation of gelatin structure can be changed from triple helices to random coil when elevating the reaction temperature above the gel melting point $\left(\sim 35^{\circ} \mathrm{C}\right)$. Regarding the influence of temperature on AuNPs at different temperature, the spherical AuNPs was synthesized with $1.5 \%(w / v)$ gelatin concentration at $\mathrm{pH} 7.0$ and temperature of $40,50,60,70,80^{\circ} \mathrm{C}$. Based on the UV-Vis data (Figure 3 and Table 2), the intensity of maximum wavelength decreased when elevating the temperature from $40^{\circ} \mathrm{C}$ to $80^{\circ} \mathrm{C}$ and the highest intensity was obtained at $40^{\circ} \mathrm{C}\left(1.14, \lambda_{\max }=529 \mathrm{~nm}\right.$, curve a). The maximum wavelength of SRP of synthesized AuNPs samples were around of 526-529 nm that indicated the formation of monodispered spherical nanoparticles (Figure 3, curve a-e). The results proved that the reaction temperature influenced directly on the production rate of the particles. Therefore, the reaction temperature of $40{ }^{\circ} \mathrm{C}$ was chosen for later investigation.

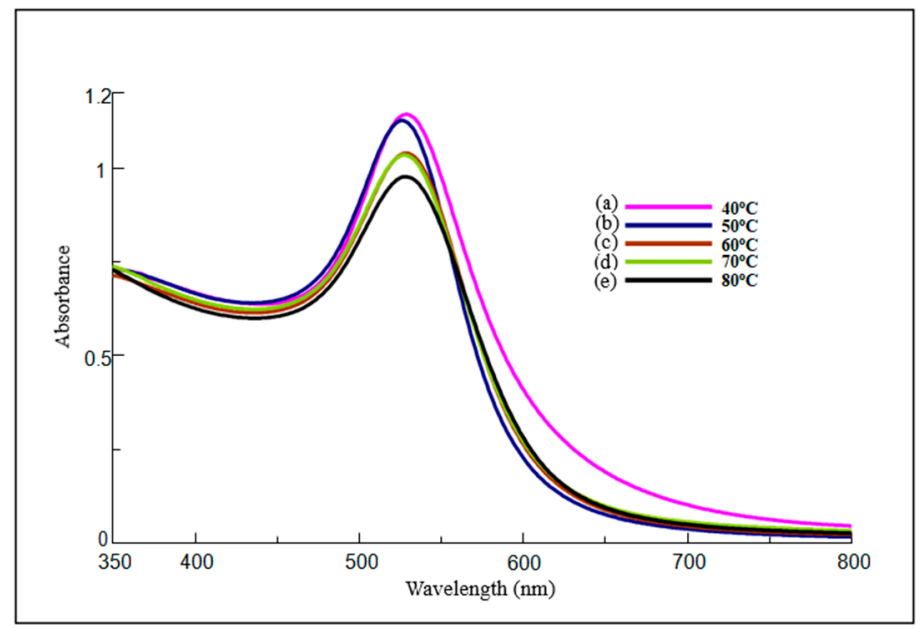

Figure 3. UV-Vis adsorption spectra of AuNPs synthesized by gelatin at different temperature of (a) 40, (b) 50, (c) 60, (d) 70 and (e) $80^{\circ} \mathrm{C}$.

Table 2. UV-Vis results of branched AuNPs synthesized by gelatin at different temperatures.

\begin{tabular}{ccc}
\hline Temperature & $\lambda \max (\mathbf{n m})$ & Absorbance \\
\hline $40^{\circ} \mathrm{C}$ & 529 & 1.144 \\
\hline $50^{\circ} \mathrm{C}$ & 526 & 1.127 \\
\hline $60^{\circ} \mathrm{C}$ & 529 & 1.040 \\
\hline $70^{\circ} \mathrm{C}$ & 528 & 1.035 \\
\hline $80^{\circ} \mathrm{C}$ & 528 & 0.977 \\
\hline
\end{tabular}

\subsection{Influence of $\mathrm{pH}$ Condition}

$\mathrm{pH}$ value also is a key factor for the growth of branched AuNPs. The rate of redox reaction may change when adjusting the $\mathrm{pH}$ value, which can be explained by the reductant forms in various $\mathrm{pH}$ conditions. In the previously suggested reduction mechanism, gold (III) chloride ion was reduced to $\mathrm{Au}^{\circ}$ atom by two reductant forms: protonated $\left(\mathrm{H}_{2} \mathrm{Asc}\right)$ and deprotonated $\left(\mathrm{HAsc}^{-}, \mathrm{Asc}^{2-}\right)$ at different $\mathrm{pH}$ conditions [15]. At $\mathrm{pH}$ value of 3.0, the shoulder band located at $703 \mathrm{~nm}$ in Figure 4 could be the spectral overlap of multiple peaks, which were corresponded to the longitudinal plasmon resonance of the elongational tips [18]. Furthermore, it is interesting to note that the gold nanodendrites obtained at pH 3.0 (Figure 5a) was not previously reported in the literature. This dendritic structure was typically achieved with mixed solution of the cationic surfactant and anionic surfactant [15]. However, to our knowledge, it is the first time gold nanodendrites are obtained with protecting agent of gelatin. At 
pH 5.0, a remarkable blue shift of absorbance peak from 608 to $553 \mathrm{~nm}$ was observed, indicating the change of AuNPs morphology (Table 3).

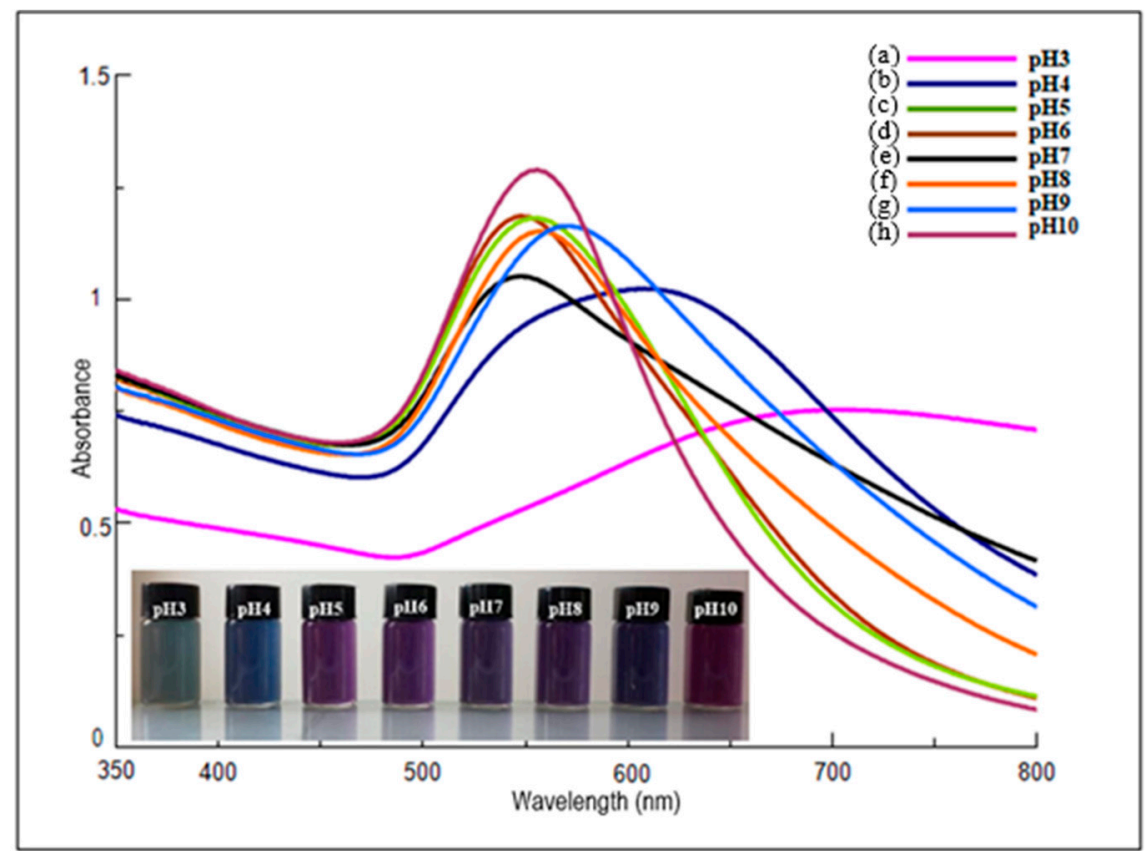

Figure 4. UV-Vis adsorption spectra of branched AuNPs synthesized by gelatin at various $\mathrm{pH}$ value: (a) $\mathrm{pH}$ 3.0; (b) $\mathrm{pH} 4.0$; (c) $\mathrm{pH}$ 5.0; (d) $\mathrm{pH}$ 6.0; (e) $\mathrm{pH} 7.0$; (f) $\mathrm{pH}$ 8.0; (g) $\mathrm{pH}$ 9.0; (h) $\mathrm{pH}$ 10.0.

Table 3. UV-Vis results of branched gold nanoparticles (AuNPs) synthesized by gelatin at various $\mathrm{pH}$ value.

\begin{tabular}{lll}
\hline pH Value & Wavelength $(\mathbf{n m})$ & Abs \\
\hline $\mathrm{pH} 3$ & 702 & 0.751 \\
\hline $\mathrm{pH} 4$ & 608 & 1.022 \\
\hline $\mathrm{pH} 5$ & 553 & 1.179 \\
\hline $\mathrm{pH} 6$ & 548 & 1.183 \\
\hline $\mathrm{pH} 7$ & 550 & 1.049 \\
\hline $\mathrm{pH} 8$ & 557 & 1.150 \\
\hline $\mathrm{pH} 9$ & 571 & 1.162 \\
\hline $\mathrm{pH} 10$ & 555 & 1.287 \\
\hline
\end{tabular}

This point was also supported by TEM images where quasi-spherical and polyhedral AuNPs with diameter ranging from 21 to $32 \mathrm{~nm}$ were observed (Figure $5 \mathrm{c}, \mathrm{d}$ ). As the $\mathrm{pH}$ value was increased to 9.0 , the absorbance peak shifted to $571 \mathrm{~nm}$ that could be featured for the morphology conversion. This red-shift also relates to both of an increase in particle size and the self-organization of gold nanoparticles. The intensity of SRP band in Figure 4 showed that the number of nanoparticles could be higher than forming at low $\mathrm{pH}$ values. According to the previous reports [19,22], this might be explained by to the viscosity and structural conformation of gelatin at various $\mathrm{pH}$ levels, which determined the electrostatic and steric interaction between gelatin and the AuNPs. Besides, the transfer of electron between gelatin and gold ions are strongly dependent on densification and structural transformation of gelatin. TEM image of branched AuNPs at pH 10.0 (Figure 6) showed that the tips of the branched structure were slightly longer and the average particles size was about $55 \mathrm{~nm}$. The zeta potential of colloidal solution was of $-5.2 \mathrm{mV}$ and electrophoretic mobility mean was $-0.4 \times 10^{-5} \mathrm{~cm}^{2} / \mathrm{Vs}$, which is 
consistent with the diffusion limited growth mechanism of branched nanoparticles. The average particle size $(74.3 \mathrm{~nm}$ ) and size distribution of branched AuNPs (gelatin concentration 2.5\%, w/v at pH 10.0) measured by DLS (Figure 6d) were slightly different from TEM images (Figure 6c). This may be due to the count of specific sizes from TEM or the layer of gelatin on the surface of nanoparticles.

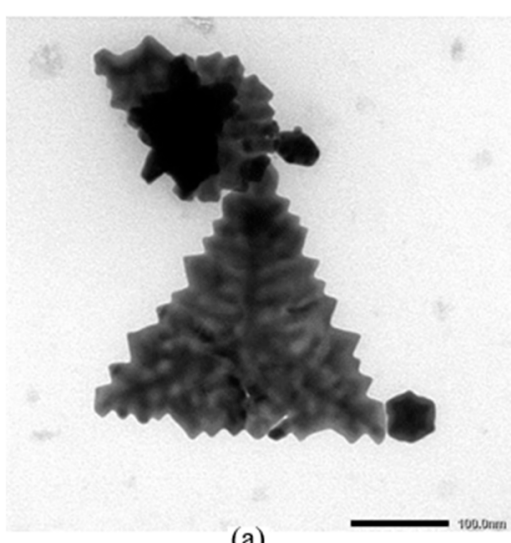

(a)

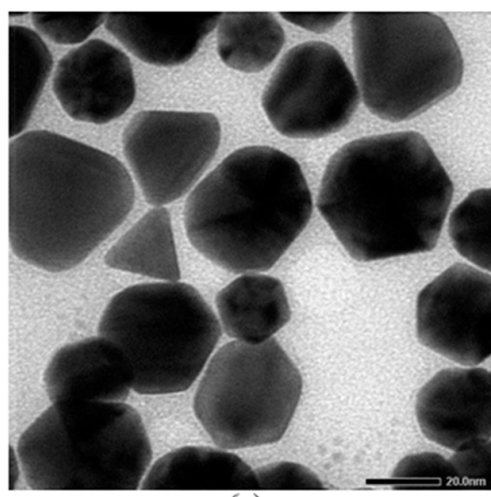

(c)

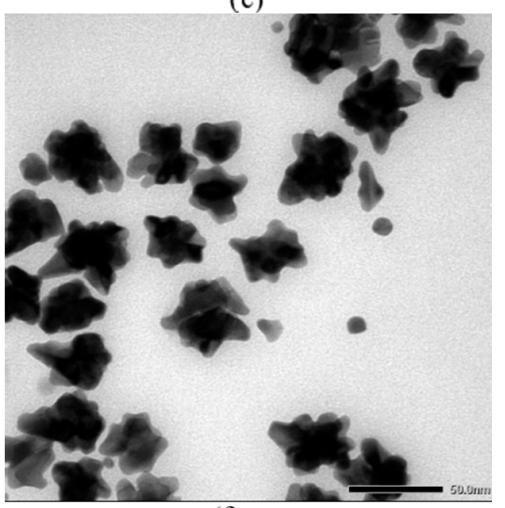

(f)

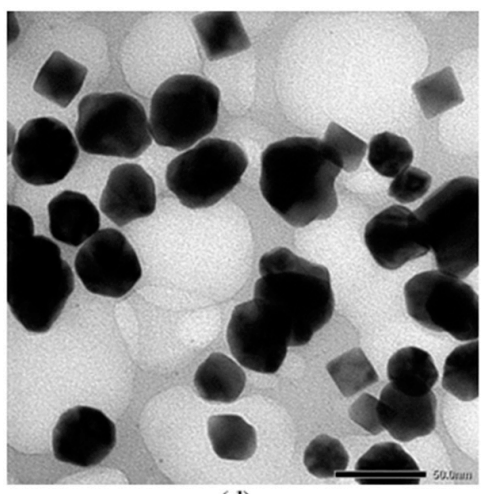

(d)

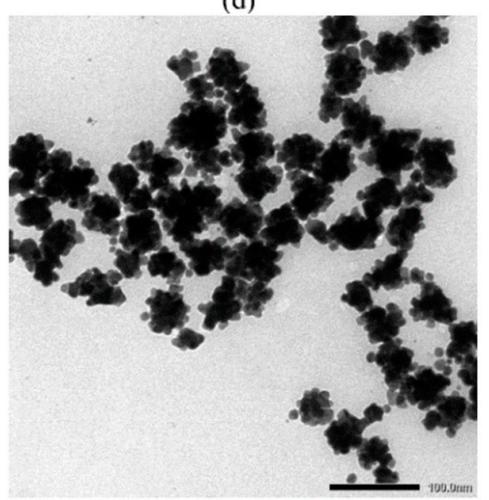

(g)

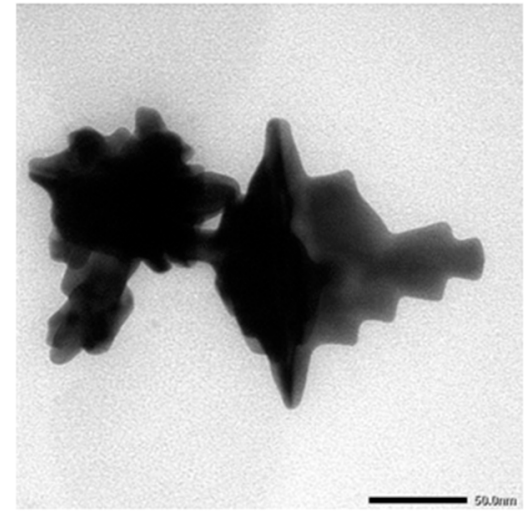

(b)

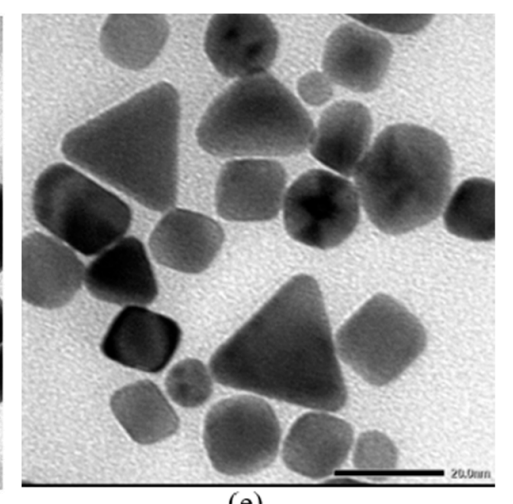

(e)

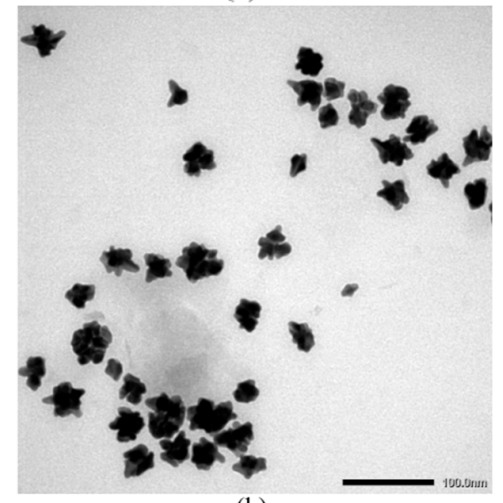

(h)

Figure 5. Representative TEM images of synthesized AuNPs at different $\mathrm{pH}$ value: (a) $\mathrm{pH} 3.0$ (scale 100 nm); (b) pH 4.0 (scale 50nm); (c) pH 5.0 (scale 20 nm); (d) pH 6.0 (scale 50 nm); (e) pH 7.0 (scale 20 nm); (f) pH 8.0 (scale $50 \mathrm{~nm}$ ); (g) pH 9.0 (scale $100 \mathrm{~nm}$ ) and (h) pH 10.0 (scale $100 \mathrm{~nm}$ ). 


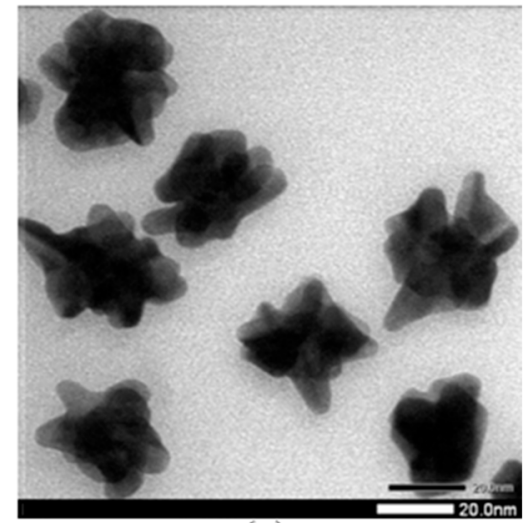

(a)

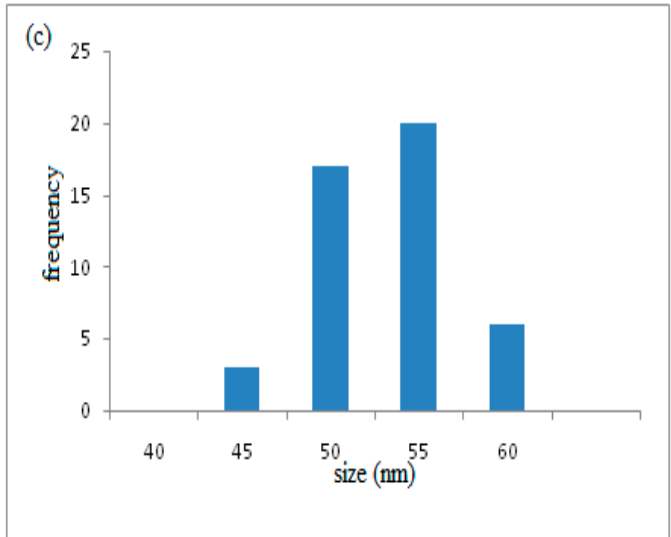

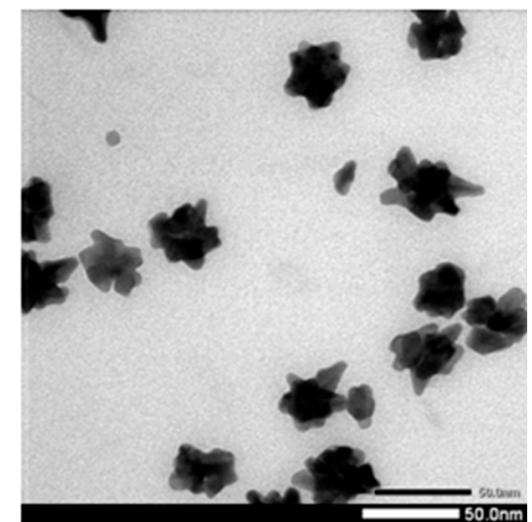

(b)

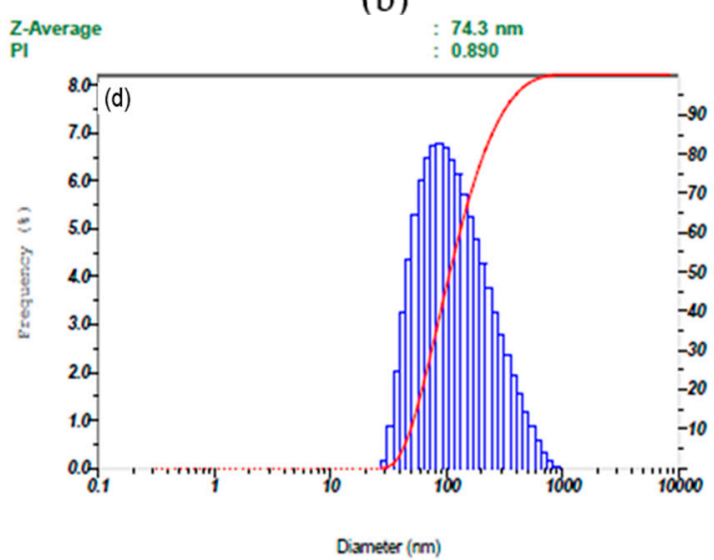

Z-Average
PI

Figure 6. TEM images branched AuNPs (a) scale $20 \mathrm{~nm}$; (b) scale $50 \mathrm{~nm}$, (c) size distribution of branched gold nanoparticles (gelatin concentration $2.5 \%, w / v$ at $\mathrm{pH} 10.0$ ) from TEM images and (d) size distribution of branched gold nanoparticles (gelatin concentration $2.5 \%, w / v$ at $\mathrm{pH} 10.0$ ) from dynamic light scattering (DLS) result.

\subsection{Proposed Mechanism of Branched AuNPs}

The growth process of gold nanoparticles could be proposed through multi-steps. These steps are: (1) the $\mathrm{AuCl}_{4}{ }^{-}$ions are reduced by ascorbic acid to generate $\mathrm{Au}^{\circ}$ atom, (2) diffusion of the pre-formed $\mathrm{Au}^{\circ}$ atom to the growth surface of gold crystal, (3) adsorption of the $\mathrm{Au}^{\circ}$ atom onto the growth surface, (4) and surface growth via the incorporation of $\mathrm{Au}^{\circ}$ atom onto the surface. Due to the limited protection of gelatin on the growth surface of Au crystal, these preformed nanoparticles can extend in other directions for branching. Interestingly, the residue of $\mathrm{Au}^{3+}$ ions in solution could be also reduced to form $\mathrm{Au}^{\circ}$ atom by the functional groups of gelatin molecules. At low $\mathrm{pH}$ value, the protonated form $\left(\mathrm{H}_{2} \mathrm{Asc}\right)$ of ascorbic acid could take place, causing the decrease of redox reaction rate and in turn the decrease of nucleation rate. Based on the diffusion-limited growth mechanism [19], when the amount of generated $\mathrm{Au}^{\circ}$ atom in solution is low due to a slow reaction rate, the growth process is regulated by the diffusion of $\mathrm{Au}^{\circ}$ atom to the particle surface. Thus, the diffusion-controlled morphologies including dendritic and branched nanoparticle are predominantly formed in colloidal solution. Besides, the disruption of intermolecular bond led to the transformation of structure from triple helices to random coils at low $\mathrm{pH}$ condition. With the coils conformation, many functional groups such as thiol and amine are exposed, facilitating their interaction with the $\{111\}$ crystalline facets [13], and resulting in the slow aggregation of $\mathrm{Au}^{\circ}$ atom onto growth surface to form nanodendrites. On the contrary, at higher $\mathrm{pH}$ value (from 5.0 to 8.0), deprotonated forms of ascorbic acid such as $\mathrm{HAsc}^{-}$and $\mathrm{Asc}^{-}$ can appear in solution, leading to the increased redox reaction rate [15]. Subsequently, the supply of growth species is also increased, and polyhedrons are formed through the crystal growth near the equilibrium state [19]. Therefore, appropriate variations of parameters such as concentration of growth 
species, viscosity of solution and reaction temperature could control the morphology conversion of gold nanoparticles. When the $\mathrm{pH}$ value of the gelatin solution reaches its isoelectric point (IEP) (PI 9.0), the gelatin dispersed in solution mainly as triple helices conformation. This caused the viscosity of solution to increase and significantly reduced the diffusion rate of growth species. The branched AuNPs was predominantly formed through the diffusion-controlled process [19]. Additionally, an increase in the solution viscosity led to a decrease in the diffusion rate of the ion $\mathrm{Au}^{3+}$, and the electrostatic interaction caused by the carboxyl and amino functional groups in molecular structure could be transformed at high $\mathrm{pH}$ condition.

\subsection{X-Ray Diffraction Analysis of Gold Nanoparticles}

From XRD pattern of the AuNPs prepared in the presence of gelatin, the diffraction peaks are observed in 2-Theta values of $38.0^{\circ}, 44.0^{\circ}, 64.5^{\circ}, 77.3^{\circ}$ (Figure 7), corresponding to $\{111\},\{200\},\{220\}$, $\{311\}$ lattice planes of the face-centered cubic ( $\mathrm{fcc}$ ) of gold crystalline structure, respectively [14]. The strongest intensity peak is assigned to plane $\{111\}$ and the broad peaks could be due to the small nanoparticle size.

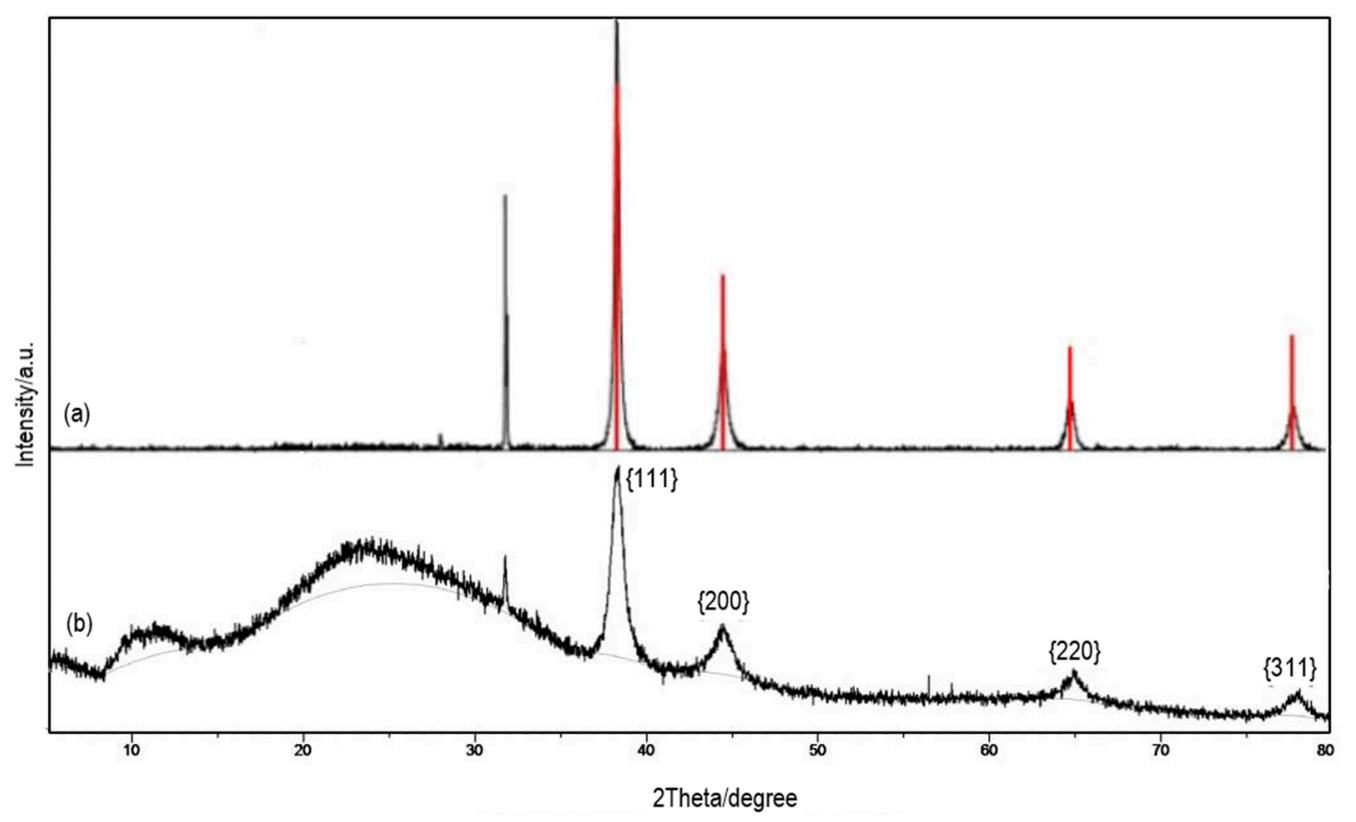

Figure 7. XRD pattern of (a) bare gold nanoparticles and (b) branched gold nanoparticles stabilized with gelatin concentration of $2.5 \%(w / v)$.

\subsection{FT-IR Analysis of Gelatin-AuNPs Interaction}

The polymeric chains of gelatin contain both of amide I, amide II and carboxyl functional groups. The molecular structure and interaction of gelatin with AuNPs were analyzed by FT-IR spectroscopy. FT-IR spectra of pure gelatin was shown Figure 8 and band positions were summarized in Table 4 . In pure gelatin spectrum (curve b), the adsorption band at 1652 is attributed to amide $\mathrm{I}$, representing the vibration of $\mathrm{C}=\mathrm{O}$ stretching. The broadening in band at $3411 \mathrm{~cm}^{-1}$ (curve b) is assigned to the $\mathrm{O}-\mathrm{H}$ vibration of retained water. The decrease of intensity peak at $3080 \mathrm{~cm}^{-1}$ (N-H stretching) (curve b) was observed in the gelatin-AuNPs spectrum (curve a), indicated the coordination of amine groups to the gold nanoparticles. N-H bending vibration of amide II groups at $1545 \mathrm{~cm}^{-1}$, in plane N-H bending coupling to C-N stretching vibrations (amide III band) at $1243 \mathrm{~cm}^{-1}$ and the $\mathrm{COO}^{-}$symmetrical stretching vibration from 1400 to $1500 \mathrm{~cm}^{-1}$ are the characteristic bands of pure gelatin (curve b) [13,23]. The bands from $1660-1650 \mathrm{~cm}^{-1}$ and $1640-1620 \mathrm{~cm}^{-1}$ are observed in $\alpha$-helix and $\beta$-antiparallel sheets, respectively [24]. When interacted with the gold nanoparticles, the amide I band at $1652 \mathrm{~cm}^{-1}$ (curve b-pure gelatin) disappeared in AuNPs-gelatin spectrum (curve a), indicating that the gelatin structure 
was changed. Additionally, the amide III band located at $1243 \mathrm{~cm}^{-1}$ (curve b) is assigned to the $\beta$-antiparallel sheets secondary structure of gelatin. Based on previous reports [24,25], $\alpha$-helix and $\beta$-antiparallel sheets secondary structure are characterized with the amide III band at 1330-1295 and $1250-1220 \mathrm{~cm}^{-1}$, respectively. The amide III band at $1243 \mathrm{~cm}^{-1}$ (curve b-pure gelatin), which was attributed to $\mathrm{C}-\mathrm{N}$ stretching vibration, disappears in spectrum of AuNPs-gelatin (curve a). Furthermore, the intensities of the amide I, amide II and carboxylic bands $\left(1500-1400 \mathrm{~cm}^{-1}\right)$ also decrease dramatically. The difference in intensity can be explained due to the blocking of vibration, corresponding to the interaction between functional groups and AuNPs. Consequently, the active sites of gelatin for binding to gold nanoparticles such as amide and carboxyclic can be determined from the FTIR results.

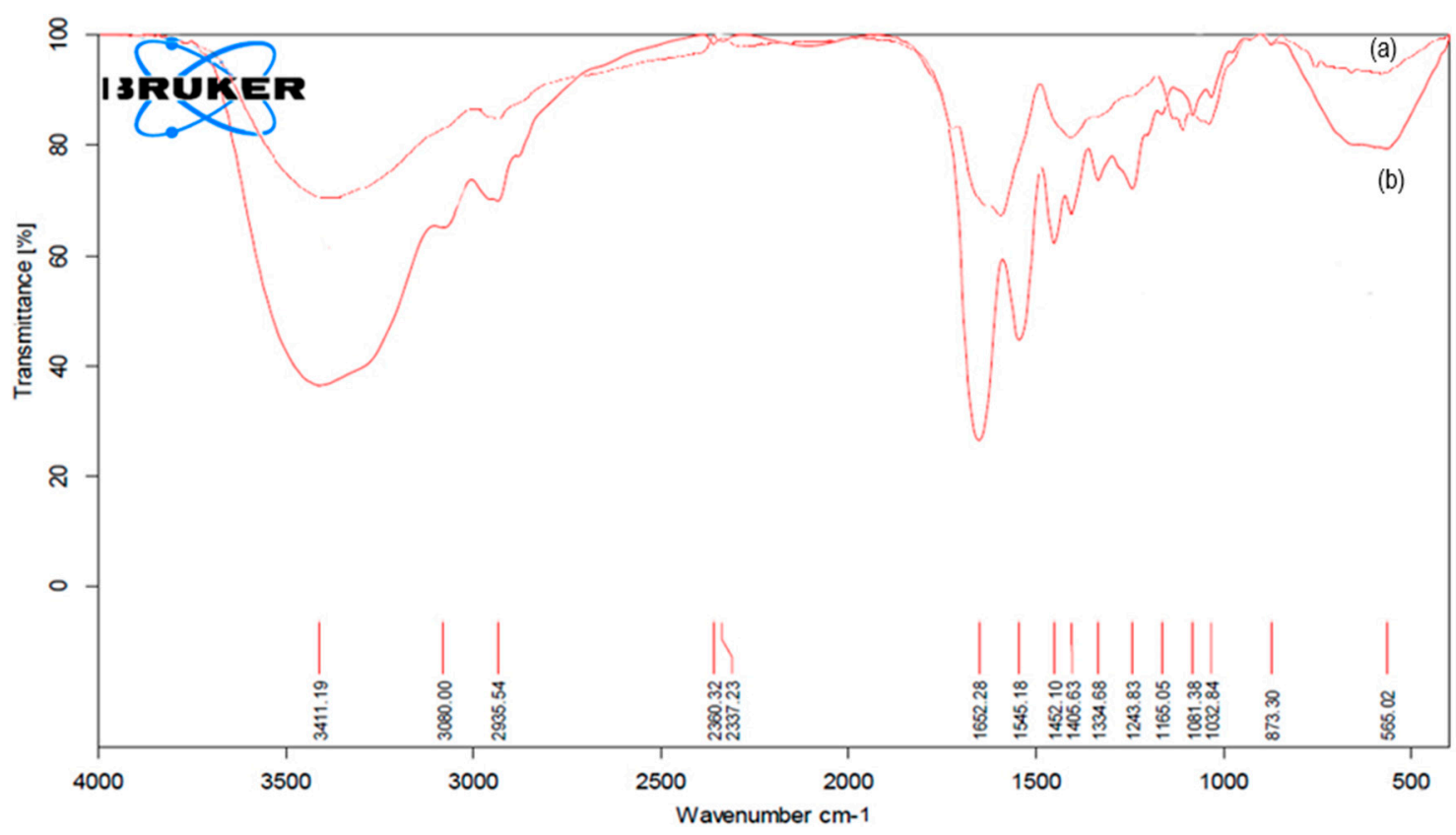

Figure 8. FTIR spectra of gelatin-AuNPs (curve a) and gelatin (curve b).

Table 4. FTIR bands position of pure gelatin and gelatin-AuNPs presented in Figure 8.

\begin{tabular}{ccl}
\hline $\begin{array}{c}\text { Position (cm } \\
\text { Pure Gelatin (Curve b) }\end{array}$ & $\begin{array}{c}\text { Position } \mathbf{( c m}^{-\mathbf{1}} \text { ) } \\
\text { Gelatin-Gold } \\
\text { Nanoparticles (Curve a) }\end{array}$ & \multicolumn{1}{c}{ Assignment } \\
\hline 3411 & 3404 & Hydrogen bonds of retain water \\
\hline 3080 & 3075 & N-H stretching \\
\hline 1652 & & Amide I in $\beta$-antiparallel sheets secondary structure \\
\hline $1640-1620$ & & Amide I in $\alpha$-helix structure \\
\hline 1545 & 1536 & Amide II N-H bending vibration \\
\hline 1243 & & $\begin{array}{l}\text { Amide III, in plane N-H bending coupling to C-N } \\
\text { stretching vibrations }\end{array}$ \\
\hline $1330-1295$ & & Amide III in $\alpha$-helix \\
\hline $1250-1220$ & & Amide III in $\beta$-antiparallel sheets \\
\hline $1500-1400$ & & COO- symmetrical stretching vibration \\
\hline
\end{tabular}

\subsection{Cell Cytotoxicity of AuNPs-Gelatin}

The cytotoxic of AuNPs-incubated human foreskin fibroblast cells was evaluated by standard SRB assay and the results were presented in Table 5 and Figure 9. After incubating for $48 \mathrm{~h}$, the growth inhibitor value (Ihn \%) of AuNPs-incubated fibroblast cell was 17.86\% (Table 5), at a dose of $100 \mu \mathrm{g} / \mathrm{mL}$ AuNPs-gelatin colloidal solution. In the case of treating with gelatin-AuNPs, Fibroblast cells 
maintained the higher survival rate than incubating with AuNPs treated cells (Table 5) at $\mathrm{pH}$ value 6.0 7.0, especially at high concentration $(100,200,400 \mathrm{ppm})$. Furthermore, when treated solely with gelatin, the viability of fibroblast cell increased to $119.46 \%$, which could be due to the presence of the natural protein. The results proved that the branched AuNPs with gelatin coating could provide highly biocompatible materials for in-vitro applications.

Table 5. Growth inhibitor value of fibroblast cells incubated with AuNPs and AuNPs-gelatin.

\begin{tabular}{cccccc}
\hline \multirow{2}{*}{ Sample } & $\begin{array}{c}\text { Concentration } \\
(\mu \mathrm{g} / \mathrm{mL})\end{array}$ & \multicolumn{4}{c}{ Growth Inhibitor Value (\%) } \\
\cline { 2 - 5 } & 400 & $\mathbf{1}$ & $\mathbf{2}$ & $\mathbf{3}$ & Average \\
\cline { 2 - 5 } Gold \\
\cline { 2 - 5 } nanoparticles & 200 & 47.69 & 42.29 & 32.50 & $40.83 \pm 7.70$ \\
\cline { 2 - 6 } & 100 & 24.62 & 16.60 & 15.36 & $18.86 \pm 5.02$ \\
\cline { 2 - 6 } & 50 & 10.77 & 13.44 & 19.64 & $14.62 \pm 4.55$ \\
\cline { 2 - 6 } & 20 & 15.38 & 14.62 & 17.86 & $15.96 \pm 1.69$ \\
\cline { 2 - 6 } & 400 & 29.62 & 29.64 & 32.14 & $30.47 \pm 1.45$ \\
\cline { 2 - 6 } AuNPs-gelatin & 200 & 18.85 & 20.55 & 25.00 & $21.47 \pm 3.18$ \\
\cline { 2 - 6 } & 100 & 21.54 & 15.81 & 16.07 & $17.81 \pm 3.23$ \\
\cline { 2 - 6 } & 50 & 18.46 & 20.95 & 17.86 & $19.09 \pm 1.64$ \\
\hline Gelatin & 20 & 7.31 & 12.25 & 13.93 & $11.16 \pm 3.44$ \\
\hline
\end{tabular}

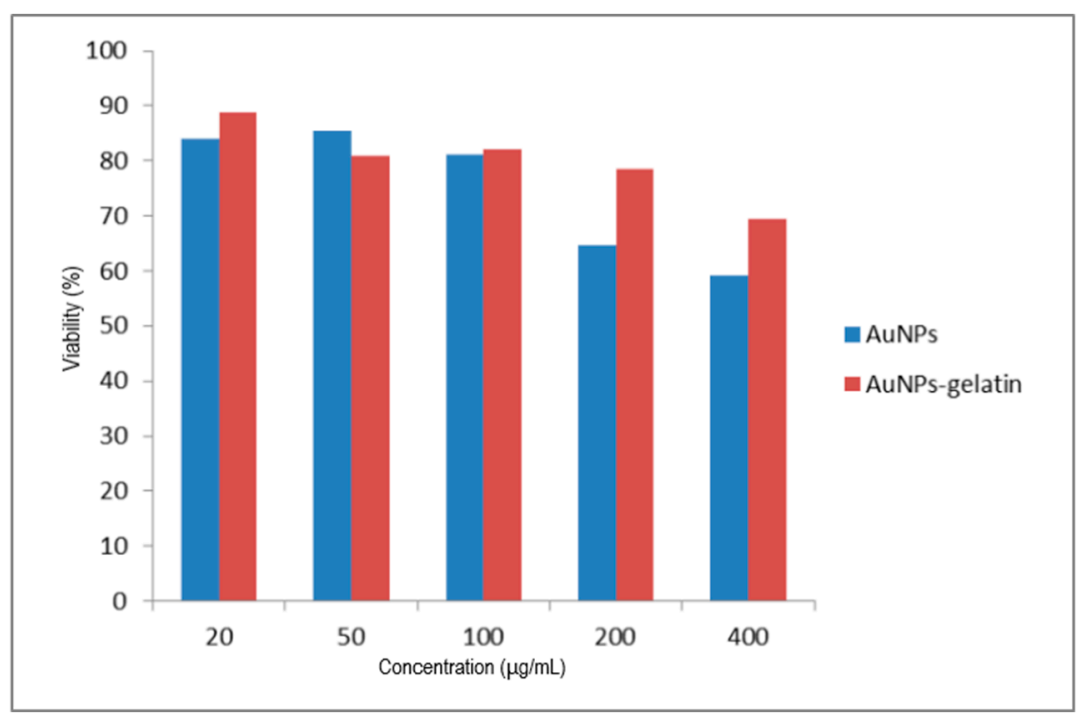

Figure 9. Cell viability of fibroblast cells treated with AuNPs and AuNPs gelatin colloidal solution after $48 \mathrm{~h}$ incubation.

\section{Conclusions}

In the present work, gold nanodendrites and gold nanostars were successfully synthesized with the natural polymer gelatin by using one-pot green synthesis method. Gelatin concentration and $\mathrm{pH}$ value play important roles in controlling the shape of gold nanoparticles. It was shown that a low $\mathrm{pH}$ value can enables the synthesis of dendrite nanoparticles with appropriate reagent ratio, while at a higher $\mathrm{pH}$ value, polygonal structures can be predominantly formed. Especially, when $\mathrm{pH}$ value of gelatin is at its IEP, branched AuNPs are synthesized. On the basic of characterization, the morphological conversion was also revealed through the proposed mechanism of branched 
nanoparticles growth. The interaction between of branched AuNPs and gelatin was also demonstrated, and the good biocompatibility of AuNPs was obtained in-vitro, testing with Human foreskin fibroblast cells. The branched AuNPs-gelatin could be a promising material to develop for future applications in drug delivery and cosmetics.

Author Contributions: Investigation, Q.K.V., M.N.N.T., P.P.N.T. and D.T.N.; Writing—original draft, Q.K.V.

Funding: This research funded by Vietnam National University HoChiMinh City (VNU-HCM) under grant number C2018-18-09.

Conflicts of Interest: The authors declare no conflict of interest.

\section{References}

1. Prashant, K.J.; Ivan, H.E.S.; Mostafa, A.E. Au nanoparticles target cancer. Nano Today 2007, 2, 18-29.

2. Parth, M.; Tapan, K.M. Recent advances in gold and silver nanoparticle based therapies for lung and breast cancers. Int. J. Pharm. 2018, 553, 483-509.

3. Li, J.J.; Wang, S.C.; Zhao, J.; Weng, G.J.; Zhu, J.; Zhao, J.W. Synthesis and SERS activity of super-multibranched $\mathrm{Au}-\mathrm{Ag}$ nanostructure via silver coating-induced aggregation of nanostars. Spectrochim. Acta A 2018, 204, 380-387. [CrossRef] [PubMed]

4. Sun, L.; Pu, S.; Li, J.; Cai, J.; Zhou, B.; Ren, G.; Ma, Q.; Zhong, L. Size controllable one step synthesis of gold nanoparticles using carboxymethyl chitosan. Int. J. Biol. Macromol. 2019, 122, 770-783. [CrossRef]

5. Canpean, V.; Gabudean, A.M.; Astilean, S. Enhanced thermal stability of gelatin coated gold nanorods in water solution. Colloids Surf. A Physicochem. Eng. Asp. 2013, 433, 9-13. [CrossRef]

6. Li, X.; Wang, Z.; Li, Y.; Bian, K.; Yin, T.; Gao, D. Self-assembly of bacitracin-gold nanoparticles and their toxicity analysis. Mater. Sci. Eng. C 2018, 82, 310-316. [CrossRef] [PubMed]

7. Razavi, S.; Seyedebrahimi, R.; Jahromi, M. Biodelivery of nerve growth factor and gold nanoparticles encapsulated in chitosan nanoparticles for schwann-like cells differentiation of human adipose-derived stem cells. Biochem. Biophys. Res. Commun. 2019, 513, 681-687. [CrossRef]

8. Jurate, V.; Rajender, S.V. Green synthesis of metal nanoparticles: Biodegradable polymers and enzymes in stabilization and surface functionalization. Chem. Sci. 2011, 2, 837-846.

9. Murphy, C.J.; Sau, T.K.; Gole, A.M.; Orendorff, C.J.; Gao, J.; Gou, L.; Hunyadi, S.E.; Li, T. Anisotropic metal nanoparticles: Synthesis, assembly, and optical application. J. Phys. Chem. B 2005, 109, 13857-13870. [CrossRef]

10. Alkilany, A.M.; Nagaria, P.K.; Hexel, C.R.; Shaw, T.J.; Murphy, C.J.; Wyatt, M.D. Cellular Uptake and Cytotoxicity of Gold Nanorods: Molecular Origin of Cytotoxicity and Surface Effects. Small 2009, 5, 701-708. [CrossRef]

11. Ali, N.; Harpinder, S.; Wayne, C.; Ryan, T.S.; Robert, R.; Mehdi, N. Gold nanorod-incorporated Gelatin-based Conductive Hydrogels for Engineering Cardiac Tissue Constructs. Acta Biomater. 2016, 41, 133-146.

12. Wang, Y.C.; Gunasekaran, S. Spectroscopic and microscopic investigation of gold nanoparticle nucleation and growth mechanism using gelatin as a stabilizer. J. Nanopart Res. 2012, 14, 1200. [CrossRef]

13. Sorina, S.; Monica, F.; Dana, M.; Simion, A. Gelatin-nanogold bioconjugates as effective plasmonic platforms for SERS detection and tagging. Colloids Surf. B. 2013, 103, 475-481.

14. Madhav, N.; Lee, S.J.; Park, I.S.; Lee, M.H.; Bae, T.S.; Kuboki, Y.; Uo, M.; Fumino, W. Synthesis of gelatin-capped gold nanoparticles with variable gelatin concentration. J. Nanopart. Res. 2011, 13, 491-498.

15. Magdalena, L.B.; Krzysztof, P.; Marek, W.; Krzysztof, F. The kinetics of redox reation of gold (III) chloride complex ions with L-ascorbic acid. Inorg. Chim. Acta 2013, 395, 189-196.

16. Sihai, C.; Zhong, L.W.; John, B.; Stephen, H.F.; David, L.C. Monopod, Bipod, Tripod, and Tetrapod Gold Nanocrystals. J. Am. Chem. Soc. 2003, 125, 16186-16187.

17. Njoki, P.N.; Lim, I.S.; Mott, D.; Park, H.; Khan, B.; Mishra, S.; Sujakumar, R.; Luo, J.; Zhong, C. Size correlation of optical and spectroscopic properties for gold nanoparticles. J. Phys. Chem. C 2007, 111, 14664-14669. [CrossRef]

18. Colleen, L.N.; Hongwei, L.; Jason, H.H. Optical Properties of Star-Shaped Gold Nanoparticles. Nano Lett. 2006, 6, 683-688.

19. Hiroaki, I. Self-Organized Formation of Hierachical Structures. Top. Curr. Chem. 2007, 270, 43-72. 
20. Damjan, P.; Sanjin, M.; Miroslav, P.; Mario, B. Role of microscopic phase separation in gelation of aqueous gelatin solution. RSC Adv. 2014, 10, 348-356.

21. Segtnan, V.H.; Isaksson, T. Temperature, sample and time dependent structural characteristics of gelatine gels studied by near infrared spectroscopy. Food Hydrocoll. 2004, 18, 1-11. [CrossRef]

22. Zhang, W.; Huang, Y.; Wang, W.; Huang, C.; Wang, Y.; Yu, Z.; Zhang, H. Influence of pH of Gelatin Solution on Cycle Performance of the Sulfur Cathode. J. Electrochem. Soc. 2010, 157, A443-A446. [CrossRef]

23. Shihua, T.; Youqun, L. Interaction via in situ binding of CdS nanorods onto gelatin. J. Colloid Interface Sci. 2011, 360, 71-77.

24. Hashim, D.M.; Man, Y.B.C.; Norakasha, R.; Shuhaimi, M.; Salmah, Y.; Syahariza, Z.A. Potential use of Fourier transform infrared spectroscopy for differentiation of bovine and porcine gelatins. Food Chem. 2010, 118, 856-860. [CrossRef]

25. Fu, F.-N.; Deoliveira, D.B.; Trumble, W.R.; Sarkar, H.K.; Singh, B.R. Secondary Structure Estimation of Proteins Using the Amide III Region of Fourier Transform Infrared Spectroscopy: Application to Analyze Calcium-Binding-Induced Structural Changes in Calsequestrin. Appl. Spectrosc. 1994, 48, 1432-1441. [CrossRef]

(C) 2019 by the authors. Licensee MDPI, Basel, Switzerland. This article is an open access article distributed under the terms and conditions of the Creative Commons Attribution (CC BY) license (http://creativecommons.org/licenses/by/4.0/). 